\title{
Der Algenbewuchs an Helgoländer Seetonnen
}

\author{
Von Kurt Beth \\ Max-Plandk-Institut für Meeresbiologie, Wilhelmshaven
}

\section{A. Einleitung}

Im folgenden sollen einige Beobachtungen über den Algenbewuchs an Helgoländer Fahrwassertonnen aus den Jahren 1938/1939 mitgeteilt werden $\left.^{1}\right)$. Sie waren durch die Notwendigkeit veranlaßt, das Verhalten bestimmter Unterwasserfarbanstriche der Praxis im Seewasser zu beurteilen und waren erweitert worden, um die Tonnen als große Versuchskörper auszunutzen, die in verschiedener Entfernung zum Felssockel Helgoland auslagen, dem einzigen Algenstandort, von dem aus sie besiedelt werden konnten. Dementsprechend sollte das Bewuchsbild besonders in Abhängigkeit vom Liegeort, wenn möglich auch von der Liegezeit zu verschiedenen Jahreszeiten erfaßt wèrden. Ökologisch sind solche Beobachtungen auch insofern noch von Interesse, als sie einen Lebensraum betreffen, der sich von jedem anderen Substrat im Gebiet um Helgoland mit einem Tidenhub von etwa $2,5 \mathrm{~m}$ durch seine mehr oder weniger gleichbleibende Höhe zum Wasserspiegel unterscheidet, und weil sie die Erstbesiedelung steriler Körper zum Gegenstand haben²).

Von den zahlreichen Untersuchungen über die Besiedelung fester Gegenstände im Meere sollen hier neben der rein floristisch orientierten Arbeit von Kirchenpauer aus dem Jahre 1862 über den Algenbewuchs an Tonnen der Elbemündung noch diejenige von MiLne (1940) genannt werden, der Tonnen des Tamars und seiner Bucht untersuchte und u. a. - den eigenen Beobachtungen bei Helgoland sehr ähnlich - das Vorkommen von drei Algenzonen feststellte, deren verschiedene Breite er zudem mit der Wassertrübe bzw. der Tageslichtabsorption gut korrelieren konnte. Alle übrigen Arbeiten befassen sich nicht näher mit Algenbewuchs an Tonnen und haben höchstens nur mittelbar Beziehungen zu den hier dargestellten Beobachtungen.

1) Bald nach Beginn der eigenen Untersuchungen, die sich auf den gesamten Bewuchs erstrecken sollten, bot Herr Dr. H. CASPERs seine Mitarbeit an und übernahm die Bearbeitung des tierischen Materials (s. ds. Bd. S. 138).

2) Hern Prof. Dr. A. Hagmeier danke ich für die Arbeitsmöglichkeit an der Biologischen Anstalt, Herrn Prof. Dr. P. Kornmann und Herrn Sahling für manchen guten Rat sowie den Herren vom Uferschutzamt für die Mitteilung der zur Untersuchung notwendigen Daten. Die in der Arbeit Caspers verwendeten Fotos stellte das Hafenbauamt freundlicherweise zur Verfügung. 


\section{B. Allgemeine Bemerkungen über die Fahrwassertonnen}

Die Tonnen um Helgoland verteilten sich auf eine Fläche von 5,5 $\times 9$ Seemeilen, unter Ausschluß der weiter entfernt liegenden Loreleybank- und Steingrund-Tonnen auf eine Fläche von 4,5 X5,5 sm (vgl. Karte auf S. 140-141). Ende 1938 wurden die Positionen einiger Tonnen verändert; in der Karte sind die neuen Orte mit einem Kreuz gekennzeichnet.

Nach dem Liegeplatz der Tonnen wurden 12 Bezirke unterschieden, die zu folgenden 3 Gebieten zusammengefaßt wurden:

Innengebiet: Gebiet der Reedetonnen (1-3);

Mittelgebiet: Tonnen des Hummerschutzgebietes und der Dünenriffe (4-8);

Außengebiet: Tonnen bis $11 \mathrm{sm}$ von der Insel entfernt (9-12).

In hydrographischer Hinsicht unterscheiden sich die einzelnen Positionen nur wenig. Der Arbeit von GoEDEcKe (1939) seien folgende Mittelwerte aus den Jahren 1927-1936 für das Oberflächenwasser entnommen:

$\begin{array}{cccccc}\text { Temperatur } & \text { Helgoland Reede } & \text { Hogstean } & \text { HSG } & \text { Sellebrunn } & \text { Düne } 0 / 2 \\ \text { Maximum } & \mathbf{1 7 , 0} & 17,4 & 17,4 & 17,4 & 17,5 \\ \text { Minimum } & \mathbf{3 , 4} & 3,0 & 2,8 & 2,7 & 2,9 \\ \text { Amplitude } & \mathbf{1 3 , 6} & \mathbf{1 4 , 4} & 14,6 & 14,7 & 14.6 \\ \text { Jahresmittel } & \mathbf{9 , 8} & \mathbf{9 , 9} & 9,9 & 9,7 & 9,9 \\ \text { Salz ehalt } & & & & & \\ \text { Maximum } & 32,99 & 33,58 & 33,09 & 33,45 & 33,51 \\ \text { Minimum } & 31,38 & 30,32 & 31,00 & 31,24 & 30,59 \\ \text { Differenz } & 1,61 & \mathbf{3 , 2 6} & 2,09 & 2,21 & 2,92 \\ \text { Jahresmittel } & 32,19 & 31,82 & 32,04 & 32,08 & 31,71\end{array}$

Ergänzend sei hinzugefügt, daß nach ZoReLL (1935) bei Steingrund im Vergleich zur Helgoländer Reede die Oberflächentemperatur durchschnittlich um $1 / 2$ Grad, höchstens um 1 Grad tiefer und der Salzgehalt um etwa $1 \%$, höchstens um 3\% niedriger war.

Bei Helgoland lagen folgende Typen eiserner Tonnen aus:

1. Leuchttonnen: Diese trugen einen Lampenstuhl und waren, gemessen von seinem Ansatz nach unten hin, 5,5-6 m lang. Sie tauchten etwa 4-5 m tief ein. Der Durchmesser betrug 2,25-3 m, der Rauminhalt 4,5-11 cbm. Ganz ähnlich gebaut waren die Leuchtheultonne Sellebrunn sowie die Glockentonne Helgoland, welche keinen Lampen-, sondern einen Glockenstuhl trug.

2. Bakentonnen: Diese hatten als Aufbau ein gitterförmiges Balkenwerk, waren ohne Aufbau fast $5 \mathrm{~m}$ lang und tauchten etwas mehr als $4 \mathrm{~m}$ in das Wasser ein. Ihr Durchmesser betrug $1,4 \mathrm{~m}$.

3. Spierentonnen: Sie waren in der Form den Bakentonnen sehr ähnlich und im wesentlichen durch einen spierenförmigen Stangenaufbau mit einem Topzeichen von ihnen unterschieden. Die Länge des eigentlichen Bojenkörpers betrug 1,1-1,9 m, die des Schwanzes etwa $1,7 \mathrm{~m}$; der Durchmesser war etwa $1,4 \mathrm{~m}$. Untergetaucht waren meistens etwa $3 \mathrm{~m}$. Ein Teil der Spierentonnen war als Stationstonnen etwa $100 \mathrm{~m}$ von den großen Tonnen entfernt zur Positionssicherung ausgelegt.

4. Spitztonnen: Die größeren Spitztonnen waren 3,5-4 $\mathrm{m}$ lang und tauchten etwa $2,5 \mathrm{~m}$ tief in das Wasser ein.

5. Buhnentonnen: Sie waren den Spitztonnen in der Form sehr ähnlich, aber wesentlich kleiner als diese.

Die Spierentonnen waren durch Budsstaben, die Spitztonnen durch Nummern bezeichnet, gleichnamige Tonnen im Norden und Süden durch den Zusatz $\mathrm{N}$ bzw. S unterschieden (z. B. $1 \mathrm{~S}$ oder B N). Die großen Leuchttonnen hießen nach ihrem Liegeort (z. B. Nathurn oder Düne 0/2) oder trugen Buchstaben (B, D, HK).

Die Tonnen waren in der üblichen Weise mit anwuchshindernden Giftfarben gestrichen. Abgesehen von den kleinen, nur mit einer einheitlichen Deckfarbe überzogenen Buhnentonnen wurden im oberen Teil sogen. Uberwasserfarben, im übrigen Teil sogen. Unterwasserfarben verwendet. Der Unterwasserfarbenanstrich war in der Beobachtungszeit bei allen Tonnen rot und gleichartig. Die Überwasserfarbanstriche waren verschiedenfarbig. Es wurde stets die gleiche Sorte weißer, roter oder schwarzer Farbe benutzt, von einigen Versuchsanstrichen abgesehen. 
In der Regel wurden die Tonnen nach einer Liegezeit von etwa einem Jahr im späten Frühjahr ausgewechselt, gereinigt und gestrichen. Die Liegezeiten schwankten jedoch zwischen 1-24 Monaten, sehr zum Vorteil der Beobachtungen, die dadurch auch zu anderen Jahreszeiten möglich waren. Eine Liste der untersuchten Tonnen mit einer graphischen Darstellung ihrer Liegezeiten befindet sich auf S. 139 dieses Bandes.

Methodisch zeichnen sich die Tonnen vorteilhaft durch ihre einfache und große Oberfläche aus. Sie wurden an Land so bald wie möglich untersucht, in der Regel innerhalb weniger Stunden nach ihrer Einbringung. Die Beobachtungen bezogen sich im wesentlichen auf die Verteilung (Deckung und Geselligkeit) der Arten, ihre Zahl, Größe und Reifestadium. Der Deckungsgrad wurde geschätzt und mit den Zahlen 1-5 gekennzeichnet. Die Geselligkeit veranschaulichen Bezeichnungen wie vereinzelt, gehäuft, gleichmäßig, dickes Band u. a. Die entnommenen Anwuchsproben wurden im Laboratorium untersucht. Sie umfaßten bei quantitativen Bestimmungen Flächen von $5 \times 5 \mathrm{~cm}$ bis zu $1 / 10 \mathrm{qm}$, deren Bewuchs mit einem Spachtel von der Tonne gelöst worden war.

Verschiedene Male wurden vom Boot aus Untersuchungen angestellt und Proben entnommen. Auf diese Weise konnte der Bewuchs über der Wasserlinie einigermaßen beobachtet werden, während über den Bewuchs der tieferen Flächen keine genauen Aussagen gemacht werden könnten.

\section{Beobachtungsergebnisse}

\section{Liste der gefundenen Arten}

Urospora isogona Batt.

Urospora bangioides Holm. \& Batt.

Urospora collabens Holm. \& Batt.

Ulothrix flaca (Dillw.) Thur.

Ulothrix implexa Kütz.

Rhizoclonium implexum (Dillw.) Batt.

Diese fädigen Grünalgen bildeten über der Wasseroberfläche ein u. U. bis เu $15 \mathrm{~cm}$ breites, z. T. sehr dickes Band. Meistens war dieses von der nach unten folgenden Enteromorphazone durch einen $Z$ wischenraum von etwa $20 \mathrm{~cm}$ getrennt, ging aber gelegentlich in diese über; nur selten kamen fädige Grünalgen fleckenweise tiefer als Enteromorpha vor. Manchmal war der Raum zwischen diesen Grünalgengürteln von einer Ectocarpuszone ausgefüllt.

Weitaus am häufigsten wurde Urospora isogona beobachtet, oft Urospora bangioides und Ulothrix flacca, gelegentlich Rhizoclonium implexum und Ulothrix implexa, sehr selten Urospora collabens. Je einmal wurde eine ganz überwiegend aus Rhizoclonium implexum bzw. aus Ulothrix flacca bestehende Zone gefunden, sonst handelte es sich um Mischbestände.

Schon nach einem Monat Liegezeit konnte sich ein Bewuchs von fädigen Grünalgen entwickelt haben. Urospora isogona war das ganze Jahr hindurch reichlich vertreten; die anderen Arten wurden nur in einigen Monaten (Frühjahr bis Herbst) beobachtet. Fädige Grünalgen kamen im ganzen Gebiet vor (vgl. Tab. 1). Die Art des Anstrichs war offenbar ohne Einfluß.

Enteromorpha compressa (L.) Grev.

Enteromorpha linza (L.) J. Ag.

Enteromorpha lingulata J. Ag.

Enteromorpha clathrata (Roth) J. Ag.

Enteromorpha (minima Naeg., micrococca Kütz.)

Enteromorpha prolifera J. Ag.

Enteromorpha sp. sp. 
Die Enteromorphen bildeten unter der Wasserlinie eine bis zu $50 \mathrm{~cm}$ breite, z. T. sehr dicke Zone, die im unteren Teil mit Punctaria, Ilea, Polysiphonia, Ceramium und anderen Algen untermischt sein konnte. Vereinzelte Enteromorpha-Pflanzen kamen bis zum unteren Ende der Tonne vor. Die beiden wichtigsten, etwa gleich häufigen Arten waren E. compressa und E. linza. Gelegentlich bildete auch E. lingulata einen wichtigen Bestandteil der Zone. Alle anderen Arten erschienen untergeordnet. Wenn man von dem Fehlen einer Zone aus E. minima und E. micrococca absieht, stimmten die Verhältnisse mit der Helgoländer Gezeitenzone überein.

Der Anwuchs von Enteromorpha trat auch während des Winters schon innerhalb der ersten beiden Monate auf, nachdem die Tonnen ausgelegt waren; im Sommer konnte sich nach 3 Monaten schon ein dichter Saum von großen Pflanzen entwickelt haben. Die Ausbildung eines geschlossenen Enteromorpha-Gürtels hängt deutlich von der Liegedauer und der Entfernung der Tonnen von der Insel ab; von zehn Tonnen des Außenbezirks hatten nur drei nach einer Liegezeit von über 9 Monaten eine Enteromorphazone.

Einen Eindruck von der Besiedelungsdichte erhält man aus einer Zählung, bei der weit über 2000 größere Enteromorpha-Pflanzen auf einer Fläche von $20 \times 20 \mathrm{~cm}$ gefunden wurden.

Die an den Tonnen gesammelten Pflanzen waren von März bis September, gelegentlich noch im Oktober fertil.

Gute Gelegenheit zu Beobachtungen über die Ansiedelung und die Wachstumsgeschwindigkeit von Enteromorpha boten im Juli und August neu ausgelegte Betonblöcke (0,2-1,6 m über $\mathrm{MNW})$ an der Helgoländer Ostmole. Die Pflanzen erreichten nach 18 Tagen bereits $2 \mathrm{~cm}$, nach 25 Tagen $7 \mathrm{~cm}$, nach 30 Tagen $9 \mathrm{~cm}$ Länge. Nach 50 Tagen waren E. lingulata $10 \mathrm{~cm}, E$. linza $21 \mathrm{~cm}$ und E. compressa $23 \mathrm{~cm}$ lang. Im Vergleich zu den Tonnen wurden die Blöcke also viel schneller besiedelt, der Anstrich mit Giftfarben verzögert den Anwuchs in der ersten Zeit merklich.

Ulva lactuca (L.) Le Jol.

Die wenigen Exemplare waren höchstens $5 \mathrm{~cm}$ groß.

Monostroma Grevillei (Thur.) Wittr.

wurde nur je einmal im März und April auf 2 Buhnentonnen gefunden.

Percursaria percursa (C. Ag.) Rosenv.

bildete auf Loreleybank Blinkt. im September 1939 ziemlich große filzuge Flächen.

Cladophora lanosa (Roth) Kütz.

Cladophora Sonderi Kütz.

Cladophora arcta (Dillw.) Kütz.

Cladophora albida (Huds.) Kütz.

Cladophora sp.

Cladophora trat nur vereinzelt und vorwiegend im mittleren Tonnenteil auf. Der Durchmesser der Cl. lanosa-Pflanzen war nicht größer als $1 \mathrm{~cm}$; Büschel von über $4 \mathrm{~cm}$ Länge wurden ubberhaupt nicht gefunden. Die Seltenheit und schlechte Entwicklung der Cladophora-Pflanzen auf den Tonnen stehen in deutlichem Gegensatz zu den Verhältnissen auf dem Helgoländer Felswatt. 
Ectocarpus tomentosus (Huds.) Lyngb.

Ectocarpus penicillatus (Ag.) Kjellm.

Ectocarpus siliculosus (Dillw.) Lyngb.

Ectocarpus confervoides (Roth) Le Jol.

Ectocarpus sp. sp.

Giffordia granulosa (Smith) Hamel

Ectocarpus-Arten wurden das ganze Jahr über an allen Tonnen gefunden. Sie siedelten sich vorwiegend im oberen Teil in der Nähe der Wasserlinie an, wurden aber in einzelnen Exemplaren gelegentlich auch reichlich im unteren Teil angetroffen. Besonders auf den Außentonnen waren sie infolge der Artenarmut und Kleinheit der anderen Formen auffällig und bedeckten z. $T$. große Flächen. Aber auch auf stark bewachsenen Tonnen kamen EctocarpusArten zwischen anderen Algen vor oder bildeten ein Band über der Enteromorpha-Zone.

Ectocarpus-Arten gehörten wie die nicht im einzelnen untersuchten Diatomeen zu den Erstbesiedlern und konnten nach 2 Monaten in sehr großer Menge (vgl. auch Tab. 2) bis ziemlich weit herunter vorhanden sein. Später wurden sie von den größeren Arten verdrängt und unterdrückt, kamen aber dann oft noch zwischen und auf größeren Formen vor. Dies war auf den Tonnen des Innengebietes mit ihrem starken Bewuchs stets der Fall. Auf den Tonnen des Außengebietes fehlte die räumliche Konkurrenz anderer Arten, innerhalb von 10 Monaten kam es dort zwar noch nicht zur Ausbildung einer geschlossenen Zone, die aber bei längerer Liegezeit an 7 von 8 Tonnen vorhanden war.

Pylaiella litoralis (L.) Kjellm.

wurde nicht häufig und nur in geringer Menge gefunden.

Sphacelaria sp.

wurde nur einmal an der Basis von Polysiphonia urceolata inmitten von Cladophora, Diatomeen, Amphipoden u. a. beobachtet.

Punctaria plantaginea (Roth) Grev.

war ein regelmäßiger und wesentlicher Bestandteil des Tonnenbewuchses, oft gehäuft und vorwiegend im oberen Teil der Tonne. Nach einjähriger Liegezeit konnte es zu einer über $10 \mathrm{~cm}$ breiten Zone kommen, die sich an die Enteromorpha-Zone nach unten anschloß. Häufiger kam Punctaria gemischt mit Enteromorpha, Rotalgen und Laminarien vor. Auf Flächen von $20 \times 20 \mathrm{~cm}$ konnten bis über 1000 Stück vorwiegend $2-5 \mathrm{~cm}$ langer Punctaria-Pflanzen gezählt werden (vgl. auch Tab. 2).

Im März wurden junge und bis zu $9 \mathrm{~cm}$ große Pflanzen gefunden, im Mai junge und alte (durchschnittlich etwa $5 \mathrm{~cm}$, maximal $19 \mathrm{~cm}$ lang), im Juni nur überständige, im Juli und im Dezember nur kleine. Kuскискs Herbarium weist im März bis $6 \mathrm{~cm}$, im April bis $18 \mathrm{~cm}$ lange Pflanzen auf, im September überständige; im März und April trugen die Pflanzen unilokuläre Sporangien. Auffällig war, daß die Alge auf 32 von insgesamt $35 \mathrm{im} \mathrm{Mai/Juni} \mathrm{untersuchten}$ Tonnen vorkam, die meistens etwa $1 \mathrm{Jahr}$ ausgelegen hatten, dagegen wurde sie nur an einer von 25 im September untersuchten Tonnen gefunden. Diese Ausnahmetonne lag im Gegensatz zu den übrigen - seit dem Frühjahr ausliegenden Tonnen - schon seit dem Herbst des vergangenen Jahres im Was- 
ser. Punctaria-Schwärmer sind also jedenfalls in den Sommermonaten praktisch nicht vorhanden. Unklar bleibt die Herkunft der kleinen Punctaria-Ptlanzen im Dezember an einer Tonne mit achtmonatiger Liegezeit und unbefriedigend die Annahme, daß sie vielleicht unterentwickelte Pflanzen der Frühjahrsgeneration darstellen.

Scytosiphon lomentaria (Lyngb.) Endl.

wurde sehr häufig im ganzen Gebiet oft gesellig und vorwiegend im mittleren Tonnenteil gefunden. Da Scytosiphon schon nach 1/1/2 Monaten sehr lang sein und außerordentlich reichlich vorkommen konnte, fiel diese Alge gerade an den nur kurze Zeit ausliegenden Tonnen mehr auf als an den stärker bewachsenen mit längerer Liegezeit. Scytosiphon ist das ganze Jahr über vorhanden.

Ilea fascia (Müll.) Fries.

wurde auf vielen Bojen des ganzen Gebiets während des ganzen Jahres vorwiegend im oberen Tonnenteil unter der Enteromorpha-Zone zusammen mit Punctaria und Rotalgen beobachtet, mitunter nur in geringen Mengen. Die Pflanzen konnten nach 2 Monaten länger als $30 \mathrm{~cm}$ sein. Sie schwärmten u. a. im März und in der Zeit von Juni-August.

Ilea fascia var. tenussima Batt.

wurde nur je einmal im Mai bzw. im September im Innengebiet gefunden.

Ilea zosterifolia (Rke.) Rosenv.

trat auf vielen Tonnen des ganzen Gebietes im oberen Teil meistens gehäuft, aber nur selten in größeren Mengen auf.

Laminaria saccharina (L.) Lamour.

bildete auf Tonnen mit längerer Liegezeit den gewichtsmäßig größten Anteil des pflanzlichen Anwuchses und kam im ganzen Gebiet vorzugsweise im mittleren Tonnenteil unter der Enteromorpha- und Punctaria-Zone vor. Im mittleren Tonnenteil waren die Pflanzen im allgemeinen länger und kräftiger als weiter unten. Entsprechend der Fortpflanzungszeit des Sporophyten, die etwa von Oktober bis Februar dauert, wurden im März nur junge Pflanzen, im Mai vorwiegend kleine, aber auch bereits Pflanzen bis zu $106 \mathrm{~cm}$ Länge gefunden. Auf der 19 Monate ausliegenden HSG 2 waren die wahrscheinlich zweijährigen Pflanzen im Juni bis zu $135 \mathrm{~cm}$ lang und $10 \mathrm{~cm}$ breit (s. Abb. 5 auf S. 148). Auf einer Fläche von $20 \times 20 \mathrm{~cm}$ der B Ltt. wuchsen am 27. 7. 38 - neben 9 Laminaria digitata (kleiner als $10 \mathrm{~cm}), 1$ Laminaria hyperborea $(3 \mathrm{~cm})$ und 107 Miesmuscheln (bis $6 \mathrm{~cm}$ groß) - 107 Laminaria saccharina mit folgenden Längen

$$
\begin{array}{cccccc}
0-10 & 11-20 & 21-30 & 31-40 & 41-50 & \mathrm{~cm} \\
19 & 42 & 36 & 7 & 3 & \text { Stück }
\end{array}
$$

Eine $2 \times 3 \mathrm{~cm}$ große mit Laminarien bewachsene Fläche auf der 14 Monate ausliegenden Nathurn Sprnt. enthielt am 25.7.38 32 Pflanzen mit folgenden Längen

$$
\begin{array}{cccccc}
0-20 & 21-40 & 41-60 & 61-80 & 81-100 \mathrm{~cm} \\
17 & 4 & 6 & 2 & 3 & \text { Stück }
\end{array}
$$

Die Charakterisierung des Bewuchses von Laminaria saccharina sei mit einem Hinweis auf die Angaben in Tab. 2 abgeschlossen. 
Laminaria digitata (Huds.) Lamour.

wurde nur gelegentlich und in viel geringerer $Z$ ahl als $L$. saccharina gefunden. Die meisten Pflanzen waren klein, und nur auf Tonne 3 Nord sowie HSG 2 und 3 wurden nach mindestens einjähriger Liegezeit einige größere Pflanzen (30-50 cm lang, $5-18 \mathrm{~cm}$ breit) gesehen.

\section{Laminaria hyperborea (Gunn.) Foslie}

war auf den Tonnen nur einmal in einem $3 \mathrm{~cm}$ langen Exemplar vertreten.

Chorda tomentosa Lyngb.

kam meistens nur in geringer $\mathrm{Zahl} \mathrm{im}$ mittleren und unteren Bojenteil vor. Die Art wurde im Mai und Juni auf Tonnen mit Liegezeiten über 11 Monaten ge funden, neben kleinen Thalli waren solche bis $122 \mathrm{~cm}$ vorhanden.

Chorda filum (L.) Stackh.

wurde nur im Mai und Juni auf Tonnen mit einer Liegezeit von mehr als 11 Monaten vereinzelt auf dem mittleren und unteren Tonnenteil einschl. der Bojenkette gefunden. Die Pflanzen waren zum Teil über $2 \mathrm{~m}$ lang.

Desmarestia viridis (Müll.) Lamour.

wurde auf vielen Tonnen, die 12 Monate und länger auslagen, im Mai und Juni gefunden. Die nie sehr zahlreichen Pflanzen waren $z$. T. länger als $30 \mathrm{~cm}$ und wühsen auf dem ganzen Tonnenkörper von der Wasserlinie bis herunter zur Ankerkette, vorzugsweise aber auf dem mittleren und unteren Tonnenteil.

Desmarestia aculeata (L.) Lamour.

wurde vereinzelt im mittleren und besonders im unteren Teil vieler Tonnen gesehen. Die Pflanzen waren im Juni bis zu $30 \mathrm{~cm}$ lang, im September bis zu $60 \mathrm{~cm}$ (auf Tonnen mit einer Liegezeit von 7-10 Monaten).

Chordaria flagelliformis (Müll.) Ag.

Von dieser. Art wurden nur einige bis $10 \mathrm{~cm}$ lange und nicht gut aussehende Pflanzen im April-Juni sowie im September auf einigen Tonnen beobachtet.

Bangia fuscopurpurea (Dillw.) Lyngb.

bildete oft große Flächen oder einen bis zu $25 \mathrm{~cm}$ breiten Gürtel zwischen der Enteromorpha- und der Urospora-Zone; sie kam auch mit fädigen Grünalgen vermischt vor. Die Art wurde im Mai-Juli, September und Dezember nur im engeren Gebiet gefunden und konnte auf vier Monate ausliegenden Tonnen reichlich vertreten sein.

Porphyra umbilicalis (L.) J..G. Ag.

kamen vorwiegend im oberen Tonnenteil auch über der Enteromorpha- und Urospora-Zone vor, gelegentlich im mittleren und unteren Teil. Die z. T. sehr großen Pflanzen wurden immer nur vereinzelt im Mai, Juli, September und Dezember angetroffen.

Porphyra linearis Grev.

wurde einmal im oberen Tonnenteil auf der 2 Monate ausliegenden Hogstean- 
Tonne im November gefunden. Die Pflanzen waren bis $z u 14 \mathrm{~cm}$ lang und in sehr gutem Zustand.

Porphyropsis coccinea (J. Ag.) Rosenv.

wuchs im Mai auf Ltt. B und D an der Kette und auf dem unteren Tonnenteil, z. T. epiphytisch auf Polysiphonia elongata, sowie auf Tonne 3 Nord in einer Probe zwischen Laminarien.

Lomentaria clavellosa (Turn.) Gaill.

war auf vielen Tonnen von der Enteromorpha-Zone abwärts vereinzelt und in geringer Zahl vertreten. Die Pflanzen kamen auch auf Seepocken und Miesmuscheln vor und erreichten höchstens $6 \mathrm{~cm}$ Länge.

Lomentaria rosea (Harv.) Thur.

war anscheinend ein wenig seltener und spärlicher als $L$. clavellosa. Die Pflanzen waren höchstens $2 \mathrm{~cm}$ groß und ganz vorwiegend auf dem unteren Tonnenteil zu finden.

Ceramium rubrum (Huds.) Ag.

ist während des ganzen Jahres bei Helgoland gemein und fruktifiziert vom Frühjahr bis zum Herbst, gelegentlich noch im Winter. Dementsprechend wurden Ceramium-Pflanzen auf fast allen Tonnen meistens schon nach kurzer Zeit gefunden. Die Ansiedlung reiner Ceramium-Bestände auf neuen Betonblöcken der Ostmole im Sommer 1939 bot Gelegenheit zu vergleichenden Zuwachsmessungen.

$$
\begin{aligned}
& \text { Liegezeit Pflanzenlänge (cm) } \\
& \begin{array}{lllllllllllllllllll}
\text { (Tage) } & 1 & 2 & 3 & 4 & 5 & 6 & 7 & 8 & 9 & 10 & 11 & 12 & 13 & 14 & 15 & 16 & 17 & 18
\end{array} \\
& 30 \text { viel }-----------
\end{aligned}
$$

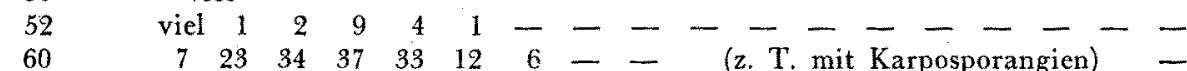

$$
\begin{aligned}
& \begin{array}{lllllllllllll}
70 & 17 & 33 & 21 & 12 & 10 & 4 & 4 & 1 & 1 & - & - & -
\end{array}- \\
& 105-\quad-512 \quad 5 \quad 13 \quad 910 \quad 4 \quad 105-2-1-1
\end{aligned}
$$

Die Pflanzen kamen in der Enteromorpha-Zone und tiefer vor, vorwiegend im oberen Tonnenteil. Sie bildeten gelegentlich ein schmales, höchstens $10 \mathrm{~cm}$ breites Band unter der Enteromorpha-Zone und verbanden dann diese zusammen mit Polysiphonia, Punctaria und Ilea mit dem Laminariabereich.

Polysiphonia urceolata (Lightf.) Grev.

ist im Sublitoral bei Helgoland gemein und fruktifiziert vorwiegend im Frühjahr und im Sommer. Die Art trat meistens im mittleren Tonnenteil unter der Enteromorpha-Zone zusammen mit Ceramium, Ilea, Punctaria und Laminaria auf. In Form einer Zone wurde P. urceolata nur einmal auf Nathurn Sprnt. im Mai nach zweijähriger Liegezeit beobachtet. Von wenigen Ausnahmen abgesehen, war $P$. urceolata auf allen genau untersuchten Tonnen mit einer Liegezeit von mehr als 5 Monaten vorhanden, mitunter auch auf Tonnen mit geringerer Liegezeit, die im Herbst untersucht werden konnten. Fruktifizierende Individuen wurden im März-Juni und im September gesehen. Die Pflanzen konnten nach 3 Monaten bis $4 \mathrm{~cm}$ und nach 12 Monaten über $10 \mathrm{~cm}$ lang sein.

Polysiphonia nigrescens (Dillw.) Grev.

war offters auf Tonnen des engeren Gebietes, aber in wesentlich geringer I Mengen als $P$. urceolata und nur einmal auf Sellebrunn Sprnt. im Mai 39 in 
größerer Menge anzutreffen. Die Pflanzen hatten auf 4 Monate ausliegenden Tonnen im September $7 \mathrm{~cm}$, nach einer Liegezeit von 7 Monaten im März $10 \mathrm{~cm}$ Länge. Im Mai wurden Karposporangien, im Juli Tetrasporangien, im September beide beobachtet.

Polysiphonia violacea (Roth) Grev.

kam vereinzelt auf manchen Tonnen vor und war auf 4 Monate ausliegenden Bojen $10 \mathrm{~cm}$ lang. Im Mai und September wurden Karpo- und Tetrasporangien sowie Antheridien gefunden.

Polysiphonia elongata (Huds.) Harv.

wurde nur in wenigen Exemplaren auf Tonnen der Binnenreede und in Klippennähe gefunden. Auf Tonnen mit einjähriger Liegezeit waren die Pflanzen über $10 \mathrm{~cm}$ lang. Sie trugen im März Antheridien und im Mai Karposporangien.

Polsiphonia atrorubescens (Dillw.) Grev.

Einige kleine Exemplare dieser auf den Dünenklippen vorkommenden Art wurden zweimal in deren Nähe auf den Bojen 2 Nord und Sellebrunn im September 1938 gefunden.

Brongniartella byssoides (Good. et Woodw.) Schmitz fand sich in geringer Zahl vorwiegend auf dem unteren Tonnenteil im Mai, Juli und September. Die Pflanzen waren nicht länger als $4 \mathrm{~cm}$.

Plocamium coccineum (Huds.) Lyngb.

kam nur in wenigen, kleinen Exemplaren im unteren Tonnenteil vor.

Delesseria alata (Huds.) Lamour.

Es wurden nur einige $1-2 \mathrm{~cm}$ lange Pflanzen auf 5 Tonnen gesehen.

Delesseria sanguinea (L.) Lamour.

Nur wenige kleine Exemplare.

Rhodomela subfusca (Woodw.) Ag.

Rhodochorton sp.

Antithamnion plumula (El1.) Thur.

Cystoclonium purpurascens (Huds.) Kütz.

Callithamnion corymbosum (Smith) Lyngb.

Von den zuletzt genannten Arten wurden nur sehr wenige, kleine Exemplare auf $1-5$ Tonnen gefunden.

\section{Tabellarische Übersichten}

In Tabelle 1 sind die Beobachtungen an 35 gründlich untersuchten Tonnen zusammengestellt, die mindestens $11 \frac{1}{2}$ Monate lang auslagen. Fast die Hälfte aller Untersuchungen entfällt auf Tonnen des Innengebietes, während in dem Mittel- und Außengebiet $10 \mathrm{bzw} .9$ Tonnen zur Verfügung standen. Für jeden der 12 Bezirke (vgl. S. 240) ist die Anzahl der untersuchten Bojen angegeben, 
auf die sich die Zahlen der Tabelle beziehen. Sie geben an, in wie vielen von den möglichen Fällen eine Art auf den Bojen eines Bezirks gefunden wurde. Bei anderer Gelegenheit festgestellte Funde wurden durch X gekennzeichnet. In den letzten vier Spalten sind die Ergebnisse jeweils gebietsweise zusammengefaßt.

Tabelle 1

Häufigkeit der Arten auf gründlich untersuchten, mindestens 111/2 Monate ausliegenden Tonnen der einzelnen Gebiete

\begin{tabular}{|c|c|c|c|c|c|c|c|c|c|c|c|c|c|c|c|c|}
\hline & 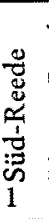 & : & 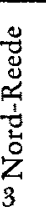 & & 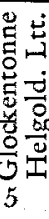 & & $\begin{array}{l}1 \\
0 \\
\\
7\end{array}$ & $\stackrel{\Xi}{8}$ & 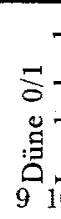 & $\frac{0}{2}$ & & & $\underset{1-3}{\stackrel{\dot{\Xi}}{\Xi}}$ & $\sum_{4-8}^{\stackrel{i}{*}}$ & $\underset{9-12}{\stackrel{5}{E}}$ & $\begin{array}{c}\stackrel{\overrightarrow{\mathrm{E}}}{\overrightarrow{\mathrm{g}}} \\
\stackrel{0}{0} \\
1-12\end{array}$ \\
\hline $\begin{array}{l}\text { Zahl der Untersuchung } \\
\text { A r t }\end{array}$ & 5 & 4 & 7 & 1 & 3 & 2 & 1 & 3 & 2 & 2 & 4 & 1 & 16 & 10 & 9 & 35 \\
\hline Fädige Grünalgen & $\mathbf{5}$ & 4 & 6 & 1 & $\mathbf{3}$ & 2 & 1 & 3 & 2 & 2 & 4 & $\mathbf{1}$ & 15 & 10 & $\mathbf{9}$ & 34 \\
\hline ora isogona & 2 & 1 & 1 & $\mathrm{x}$ & 3 & $\mathrm{x}$ & 1 & 1 & 1 & 1 & 1 & 1 & 4 & 5 & 4 & 13 \\
\hline Urospora bangioides & $\mathrm{x}$ & 2 & $\mathbf{x}$ & $x$ & 1 & 0 & 0 & 0 & 0 & 0 & 0 & 0 & 2 & 1 & 0 & $\therefore$ \\
\hline rospora collabens & 0 & 0 & $\mathrm{x}$ & 0 & 0 & 0 & 0 & 0 & 0 & 0 & 0 & 0 & $\mathrm{x}$ & $\mathrm{x}$ & 0 & \\
\hline Ulothrix implexa & 2 & $\mathrm{x}$ & $\mathrm{x}$ & 0 & 0 & 0 & 0 & 0 & 0 & 0 & 1 & 0 & 2 & 0 & 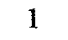 & \\
\hline Ulathrix flacca & 1 & $\mathrm{x}$ & $\mathrm{x}$ & 0 & 0 & $\mathrm{x}$ & 0 & 0 & 0 & 0 & $\mathrm{x}$ & 0 & 1 & $\mathrm{x}$ & $\mathrm{x}$ & \\
\hline Rhizoclonium implexum & $\mathrm{x}$ & 0 & $\mathbf{x}$ & 0 & 0 & $x$ & 0 & 0 & 0 & 0 & $x$ & 0 & $\mathrm{x}$ & $\mathrm{x}$ & $\mathrm{x}$ & $\mathrm{x}$ \\
\hline norpha compressa & $\mathbf{5}$ & $\mathbf{3}$ & 7 & $\mathbf{1}$ & 3 & 2 & $\mathbf{1}$ & 3 & 2 & $\mathbf{1}$ & 4 & 1 & 15 & 10 & 0 & 33 \\
\hline Enteromorpha linza & 4 & 4 & 7 & 1 & 3 & 2 & $\mathbf{1}$ & $\mathbf{3}$ & 2 & 2 & $\mathbf{3}$ & & 15 & 10 & $\varepsilon$ & 33 \\
\hline nteromorpha lingulata & 1 & 2 & I & 0 & 0 & 0 & 0 & 0 & 0 & $\mathrm{x}$ & 2 & ( & 4 & 0 & & \\
\hline interomorpha prol & 0 & $x$ & 0 & 0 & 0 & 0 & 0 & i & 0 & 0 & 0 & & $\mathrm{x}$ & 0 & & $x$ \\
\hline Lorpha clat & $\mathbf{x}$ & 0 & 0 & 0 & 0 & 0 & 0 & & & 0 & 0 & & $\mathbf{x}$ & 0 & & \\
\hline orpha mini & 1 & 0 & 1 & 0 & 0 & 0 & 0 & 0 & & 0 & 0 & & 2 & 0 & & 2 \\
\hline Ulv & 0 & 1 & 1 & 0 & 0 & 0 & 0 & 0 & 0 & 0 & 0 & & 2 & 0 & & 3 \\
\hline a Grev & 0 & $\mathrm{x}$ & 0 & 0 & 0 & 0 & 0 & 0 & 0 & 0 & 0 & & $\mathrm{x}$ & 0 & 0 & 12 \\
\hline ia percursa & 0 & 0 & 0 & 0 & 0 & 0 & 0 & & ( & $\mathrm{x}$ & 0 & & 0 & 0 & & $x$ \\
\hline ora sn & 5 & 3 & 5 & 1 & 0 & 2 & 1 & & & 0 & 0 & & 13 & 7 & & 22 \\
\hline us sp. & $\mathbf{5}$ & 4 & 3 & 1 & 3 & 2 & $\mathbf{1}$ & & 2 & 2 & 4 & & 12 & 10 & & 31 \\
\hline to & 1 & 1 & 4 & 0 & 1 & 0 & 0 & 1 & 0 & 1 & 1 & & 6 & 2 & & 11 \\
\hline Is silicu & 0 & 0 & $\mathrm{~m}$ & 0 & 0 & 0 & 0 & 0 & 0 & 0 & 0 & & $\mathrm{x}$ & 0 & & $\mathrm{x}$ \\
\hline r & 0 & $\mathrm{x}$ & 0 & 0 & 0 & 0 & 0 & 0 & 0 & 0 & 1 & & $\mathrm{x}$ & 0 & & \\
\hline$s$ fasc & 0 & 0 & $\mathrm{x}$ & 0 & 0 & 0 & 0 & & 0 & 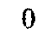 & 0 & & $\mathrm{x}$ & 0 & & \\
\hline G & 2 & 2 & 3 & & 1 & 0 & 1 & 1 & 1 & 1 & 1 & & 7 & 3 & & 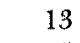 \\
\hline lito & 1 & $\mathrm{x}$ & 2 & 0 & $x$ & 0 & 0 & c & 1 & 0 & 1 & & 3 & $\mathrm{x}$ & & \\
\hline & 1 & 0 & 0 & 0 & 0 & 0 & 0 & 0 & 0 & 0 & 0 & 0 & 1 & 0 & 0 & \\
\hline & $\mathbf{5}$ & 4 & 7 & 1 & 3 & 2 & 1 & 3 & 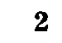 & 2 & $\mathbf{3}$ & & 16 & 10 & 8 & 34 \\
\hline $\mathbf{n}$ & 4 & $\mathbf{3}$ & 6 & $\mathbf{1}$ & 3 & 1 & $\mathbf{x}$ & 3 & 2 & 2 & 3 & & 13 & 8 & 8 & 29 \\
\hline I & 2 & $\mathbf{3}$ & 4 & $\mathbf{1}$ & $\mathbf{2}$ & 0 & $\mathbf{x}$ & 2 & 2 & 1 & 2 & $\mathbf{x}$ & 9 & 5 & 5 & I. \\
\hline erif & 1 & 1 & $\mathbf{3}$ & $\mathbf{1}$ & $\mathbf{1}$ & 1 & $\mathbf{x}$ & 1 & 2 & 2 & 1 & 0 & $\mathbf{5}$ & 4 & 5 & \\
\hline & $\mathbf{5}$ & 4 & 7 & 1 & $\mathbf{3}$ & 2 & 1 & 3 & 2 & 2 & 4 & $\mathbf{x}$ & 16 & 10 & 8 & 34 \\
\hline & $\mathrm{x}$ & 1 & 3 & 1 & 0 & 0 & 1 & 1 & 0 & 0 & 1 & . & 4 & 3 & $a$ & \\
\hline & $\mathrm{x}$ & 0 & 0 & 0 & 0 & 0 & 0 & 0 & 0 & 0 & 0 & 0 & $\mathrm{x}$ & 0 & 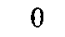 & \\
\hline tomer & 1 & 2 & 5 & 1 & 1 & 1 & 0 & $\mathbf{0}$ & 2 & 1 & 0 & 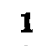 & 8 & 3 & $x$ & \\
\hline a filum & 1 & 2 & 3 & 0 & 2 & 1 & 1 & 0 & 0 & 0 & 0 & 0 & 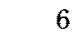 & 4 & 0 & \\
\hline & 5 & 2 & 4 & 1 & 2 & 2 & o & 1 & 2 & 1 & 1 & 0 & 11 & 6 & 4 & 2 \\
\hline & 2 & $\overline{3}$ & 1 & 1 & 1 & 1 & 0 & 1 & 0 & 0 & 0 & I & 6 & 4 & 1 & 1 \\
\hline & 0 & 0 & 0 & 1 & 1 & 0 & 0 & 1 & 0 & $\mathrm{x}$ & 0 & 0 & $x$ & 3 & $x$ & \\
\hline & 3 & 0 & 5 & 1 & 0 & 0 & 1 & 0 & 0 & 0 & 0 & 0 & 8 & 2 & 0 & 1 \\
\hline & 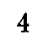 & 3 & 5 & 1 & 2 & 0 & 0 & 0 & 0 & 1 & 0 & & 12 & 3 & 1 & 16 \\
\hline ra & a & 0 & 0 & 1 & 0 & 1 & 0 & 0 & 0 & 0 & 0 & 0 & 0 & 2 & 0 & \\
\hline
\end{tabular}




$\begin{array}{llll}\text { Porphyropsis coccinea } & 0 & \mathbf{1} & \mathbf{1} \\ \text { Polysiphonia urceolata } & \mathbf{5} & \mathbf{4} & \mathbf{7} \\ \text { Polysiphonia nigrescens } & 3 & 3 & 6 \\ \text { Polysiphonia violacea } & 0 & 1 & 2 \\ \text { Polysiphonia elongata } & 2 & 2 & 3 \\ \text { Plocamium coccineum } & 0 & 0 & \mathbf{x} \\ \text { Ceramium rubrum } & \mathbf{2} & 2 & 1 \\ \text { Polysiphonia atrorubescens } & \mathbf{5} & \mathbf{4} & \mathbf{7} \\ \text { Brongniartella byssoides } & 0 & 3 & 1 \\ \text { Lomentaria rosea } & 4 & 1 & 6 \\ \text { Lomentaria clavellosa } & \mathbf{4} & \mathbf{2} & \mathbf{5} \\ \text { Delesseria sanguinea } & 1 & 1 & 1 \\ \text { Delesscria alata } & 0 & 2 & 2 \\ \text { Rhodomela subfusca } & 0 & 1 & 0 \\ \text { Rhodochorton } & 1 & 0 & 0\end{array}$

$\begin{array}{lllll}0 & 0 & 0 & 0 & 0 \\ \mathbf{1} & \mathbf{3} & \mathbf{2} & \mathbf{1} & \mathbf{3} \\ \mathbf{1} & 0 & 1 & \mathbf{x} & 2 \\ 0 & \mathbf{1} & \mathbf{x} & \mathbf{x} & \mathbf{x} \\ 0 & 0 & 0 & 0 & 0 \\ 0 & 0 & 0 & 0 & 0 \\ 1 & 2 & 1 & 0 & 2 \\ \mathbf{1} & \mathbf{3} & \mathbf{2} & \mathbf{1} & \mathbf{3} \\ 0 & 0 & 0 & 0 & 0 \\ 0 & \mathbf{2} & 0 & \mathbf{x} & \mathbf{x} \\ \mathbf{1} & \mathbf{3} & \mathbf{1} & \mathbf{x} & \mathbf{3} \\ 0 & 0 & 0 & 0 & 1 \\ 0 & 0 & 0 & 0 & 1 \\ 0 & 1 & 0 & 0 & 1 \\ 0 & 0 & 0 & 0 & 0\end{array}$

$\begin{array}{llll}0 & 0 & 0 & 0 \\ \mathbf{2} & \mathbf{2} & \mathbf{3} & \mathbf{1} \\ 0 & 0 & 0 & 0 \\ 0 & \mathbf{x} & 0 & 0 \\ 0 & 0 & 0 & 0 \\ 0 & 0 & 0 & 0 \\ 1 & 0 & 0 & 0 \\ \mathbf{2} & \mathbf{2} & \mathbf{1} & \mathbf{1} \\ 0 & 0 & 0 & 0 \\ 2 & 1 & 0 & 0 \\ \mathbf{2} & \mathbf{2} & \mathbf{2} & \mathbf{0} \\ 0 & 0 & 0 & 0 \\ 0 & 0 & 0 & 0 \\ 0 & 0 & 0 & 0 \\ 0 & 0 & 0 & 0\end{array}$

$\begin{array}{rrrr}2 & 0 & 0 & 2 \\ \mathbf{1 6} & \mathbf{1 0} & \mathbf{8} & \mathbf{3 4} \\ \mathbf{1 2} & 4 & 0 & 16 \\ 3 & 1 & \mathbf{x} & \mathbf{4} \\ 7 & 0 & 0 & \mathbf{7} \\ \mathbf{x} & 0 & 0 & \mathbf{x} \\ 5 & 6 & 1 & 12 \\ \mathbf{1 6} & \mathbf{1 0} & \mathbf{6} & \mathbf{3 2} \\ \mathbf{4} & 0 & 0 & 4 \\ 11 & 2 & \mathbf{3} & \mathbf{1 6} \\ \mathbf{1 1} & \mathbf{8} & \mathbf{6} & \mathbf{2 5} \\ 3 & 1 & 0 & 4 \\ 4 & 1 & 0 & 5 \\ 1 & 2 & 0 & 3 \\ 1 & 0 & 0 & \mathbf{1}\end{array}$

Liest man die Kolonnen in vertikaler Richtung, so vermitteln sie ein Bild der "Lokalflora“ an den Tonnen der einzelnen Gebiete. In horizontaler Richtung gelesen, zeigen sie die gebietsmäßige Verbreitung einer Art an. Die Arten, die ziemlich regelmäßig an allen Tonnen anzutreffen sind, wurden durch fetten Drurk hervorgehoben. Im Vergleich zu diesen können die übrigen Arten als Begleitformen betrachtet werden, selbst wenn sie, wie z. B. Polysiphonia nigrescens, P. elongata, Lomentaria rosea, Porphyra, Bangia und Chorda filum auf den Tonnen des Innengebietes verhältnismäßig oft auftraten.

Eine subjektive Schätzung des mengenmäßigen Vorkommens der am häufigsten angetroffenen Arten bringt Tab. 2. Wie zu erwarten, wird die Menge des Anwuchses geringer, je weiter die Tonnen von der Insel entfernt liegen. Eine Ausnahme bildet Ectocarpus, der auf den entfernteren Tonnen reichlicher vorkam; die Gründe dafür wurden bereits früher (S. 243) erörtert.

Die Verteilung der Arten auf den verschieden tief eintauchenden Abschnitten des Tonnenkörpers wird in Tab. 3 dargestellt. Den Angaben liegen die Beobachtungen an 10 Tonnen zugrunde; die Zahlen zeigen an, in wie vielen Fällen eine Art im oberen, mittleren oder unteren Teil angetroffen wurde. Nur wenige Arten sind ausschließlich auf eine Zone beschränkt. So siedeln sich die fädigen Grünalgen. Enteromorpha lingulata. Ilea zosterifolia und Bangia nur im oberen Teil, Desmarestia aculeata und Lomentaria rosea nur im unteren Teil der Tonnen an. Die meisten Arten sind in zwei oder allen drei Zonen vertreten, wenn auch einer der Abschnitte bevorzugt wird (durch fetten Druck gekennzeichnet). Man muß natürlich auch den mengenmäßigen Anteil der Arten in den verschiedenen Tonnenabschnitten berücksichtigen. Die auf der ganzen Höhe der Tonne vorhandenen Enteromorphen können im obersten Teil unter der Wasserlinie eine gürtelförmige Zone bilden, an die sich ein geschlossenes Band von Ceramium anschließen kann. Auch Ectocarpus und Punctaria wuchsen vorwiegend im oberen Teil der Tonnen. Laminaria saccharina herrschte mengenmäßig im mittleren Tonnenteil vor, wenn sie auch in den beiden anderen Zonen nicht fehlte. Im übrigen war die Verteilung der Arten auf dem Tonnenkörper von vielen Zufälligkeiten mitbestimmt, so daß sich besonders für die nicht so häufigen Formen keine Regelmäßigkeiten in der Verteilung erkennen lassen. Oftmals siedelten sich die Algen auf unregelmäßigen Flächen von verschiedener Größe an, wie dies ganz ähnlich auch bei der tierischen Besiedelung beobachtet wurde (vgl. Caspers, S. 153). Diese Unregelmäßigkeiten machten eine objektive mengenmäßige Erfassung des Bewuchses leider nicht möglich (vgl. Tab. 2). 


\section{Tabelle 2}

Mengenmäßige Schätzung des pflanzlichen Bewuchses von neun im Mai 1939 untersuchten Tonnen nach 12monatiger Liegezeit

\begin{tabular}{|c|c|c|c|c|c|c|c|c|c|}
\hline \multirow[b]{2}{*}{ Art Tonne } & \multicolumn{3}{|c|}{ Innengebiet } & \multicolumn{2}{|c|}{ Mittelgebiet } & \multicolumn{4}{|c|}{ Außengebiet } \\
\hline & $\begin{array}{c}\text { D Ltt. } \\
1) \\
\end{array}$ & C Süd & $\begin{array}{c}\mathrm{BN} \\
2)\end{array}$ & $\begin{array}{c}\text { Hlgd } \\
\text { Itt. } \\
1)\end{array}$ & $\begin{array}{c}\text { Düne } \\
0 / 2 \\
\text { Sprnt. }\end{array}$ & $\begin{array}{c}\text { Düne } \\
0 / 1\end{array}$ & $\begin{array}{l}\text { Lor:- } \\
\text { ley } \\
\text { Bkt. } \\
\end{array}$ & \begin{tabular}{|c} 
Stgrd \\
S \\
Sprnt.
\end{tabular} & $\begin{array}{c}\text { Stgrd } \\
\mathrm{N}\end{array}$ \\
\hline Fädige Grünalgen & $w-m$ & Zone & Zone & $(\mathrm{m})$ & $\mathrm{m}$ & $(\mathrm{m})$ & Zone & $\mathrm{m}$ & $\mathbf{w}$ \\
\hline Enteromorpha compr. u. linza & $\mathrm{m}$ & $\mathrm{m}$ & $\mathrm{m} \cdot \mathrm{v}$ & $\mathrm{v}$ & $\mathrm{m}$ & sw & sw & $(\mathrm{s}) \mathrm{w}$ & sw \\
\hline Cladophora sp. sp. & + & - & - & - & t & + & - & $\longrightarrow$ & - \\
\hline Ectocarpus sp. sp. & + & + & $\mathrm{m} \cdot \mathrm{v}$ & $\mathrm{m} \cdot \mathrm{v}$ & $\mathrm{v}$ & $s v$ & $\mathrm{~m}$ & sv & sv \\
\hline Laminaria saccharina & $\mathrm{V}-\mathrm{sv}$ & $(\mathrm{m} \cdot \mathrm{v})$ & $\mathbf{v}$ & $\mathrm{m}$ & $w$ & $\mathrm{~V} * \mathrm{~s} \mathrm{~V}$ & sw & sw & sw \\
\hline Punctaria plantaginea & $\mathrm{m}$ & $\mathrm{m}$ & $\mathrm{m}$ & $\mathrm{v}$ & $w$ & $\mathrm{~m}$ & + & + & + \\
\hline Scytosiphon lomentaria & + & sw & $w$ & + & + & w.m & sw & + & + \\
\hline Ilea fascia & $\mathrm{m}$ & - & $\mathrm{m}$ & $\mathrm{m}-\mathrm{v}$ & $w$ & + & sw & $一$ & - \\
\hline Ilea zosterifolia & - & - & - & $\mathrm{m}-\mathrm{v}$ & 十. & + & (m) & - & - \\
\hline Desmarestia viridis & m & sw & + & + & - & + & - & 一 & - \\
\hline Desmarestia aculeata & + & 一 & - & - & 一 & - & - & - & + \\
\hline Chorda filum & $\mathrm{m}$ & $\mathbf{w}$ & + & + & 一 & + & - & - & - \\
\hline Chorda tomentosa & $\mathrm{m}$ & - & - & $t$ & - & + & - & - & 1 \\
\hline Polysiphonia urceolata & $\mathrm{v}$ & $\mathbf{m}$ & $\mathrm{v}$ & (w) & $\mathrm{m}$ & $w$ & sw & sw & + \\
\hline Ceramium rubrum & $\mathrm{v}$ & m & $v$ & $(w)$ & $w$ & $w$ & sw & - & + \\
\hline Lomentaria clavellosa & $\mathrm{v}$ & + & $s w$ & + & + & + & + & + & - \\
\hline Lomentaria rosea & - & + & - & + & + & + & + & - & - \\
\hline Porphyra umbilicalis & w & - & + & + & - & - & - & - & - \\
\hline Brongniartella byssoides & + & - & - & + & 一 & + & - & 一 & - \\
\hline Delesseria alata & + & $一$ & + & 一 & 一 & + & 一 & - & - \\
\hline Polysiphonia nigresc. u. elong. & - & - & + & - & - & - & - & - & - \\
\hline Polysiphonia violacea & + & - & - & - & - & - & - & - & 一 \\
\hline $\begin{array}{l}+=\text { vorhanden } \\
\text { sw }=\text { sehr wenig }\end{array}$ & & $\begin{array}{l}\mathrm{w}= \\
\mathrm{m}=\end{array}$ & $\begin{array}{l}\text { venig } \\
\text { nittel }\end{array}$ & & & & $\begin{array}{l}\mathrm{v}=\mathrm{v} \\
\mathrm{v}=\mathrm{sc}\end{array}$ & hr viel & \\
\hline
\end{tabular}

1) Tonnenkörper mit Versuchsfarben gestrichen.

2) Tonne wurde im September 1938 nördlich verzogen, blieb aber auf der Nordreede.

Tabelle 3

Besiedelung des oberen (I), mittleren (II) und basalen (III) Teils von 10 Tonnen

\begin{tabular}{|c|c|c|c|c|c|c|}
\hline & $I$ & II & III & & I & II \\
\hline Fädige Grünalgen & 9 & 0 & 0 & Desmarestia viridis & 1 & 2 \\
\hline Enteromorpha compr. & 9 & 5 & 4 & Bangia fuscopurpurea & $\mathbf{3}$ & 0 \\
\hline Enteromorpha linza & 10 & 10 & 7 & Porphyra & $\mathbf{5}$ & 1 \\
\hline Enteromorpha lingul. & 4 & 0 & 0 & Porphyropsis cocc. & 0 & 0 \\
\hline Ulva lactuca & 1 & 0 & 0 & Ceramium rubrum & 7 & 8 \\
\hline Cladophora sp. & 3 & 6 & 2 & Polysiphonia urceol. & 9 & 8 \\
\hline Ectocarpus sp. & 8 & 7 & 4 & Polysiphonia nigresc. & 1 & 3 \\
\hline Punctaria plantag. & 7 & 8 & 5 & Polysiphonia violacea & 0 & 1 \\
\hline Ilea fascia & 6 & 6 & 1 & Polysiphonia elongata & 1 & 1 \\
\hline Ilea zosterifolia & 3 & 0 & 0 & Lomentaria clavell. & 3 & 7 \\
\hline Chorda & 0 & 4 & 5 & Lomentaria rosea & 0 & 0 \\
\hline Laminaria saccharina & 6 & 8 & 6 & Brongniartella byss. & 0 & 2 \\
\hline Scytosiphon loment. & 6 & 9 & 7 & Plocamium coccineum & 0 & 0 \\
\hline Desmarestia aculeata & 0 & 0 & 4 & Delesseria sanguinea & 0 & 0 \\
\hline
\end{tabular}


Ein Vergleich des Bewuchses von Tonnen gleicher Position und Liegezeit in den beiden aufeinanderfolgenden Untersuchungsjahren zeigte im Auftreten und in der Menge seltener und gemeiner Arten teils große Ähnlichkeiten, teils aber auch beträchtliche Verschiedenheiten, die nur als zufällig erklärt werden können und daher nicht näher besprochen werden sollen.

Die Wirkung der Farbanstriche war bei dem relativ kleinen Material infolge der beträchtlichen Fluktuationen schwer zu erkennen und abzuschätzen. Sicherlich verhindert das Farbgift die Besiedelung während einer gewissen Zeit, welche nach meinen Beobachtungen an den Tonnen und an besonderen Probeplatten höchtens ein halbes Jahr, in der Regel aber viel weniger ausmacht. Die mit Unterwasserfarbe gestrichenen Flächen wurden von den Algen offenbar später bewachsen als die mit Überwasserfarbe gestrichenen, und einige Beobachtungen deuteten darauf hin, daß die schwarzen Tonnen am ehesten, die roten am spätesten bewuchsen, daß also die verschiedene Farbe des Überwasseranstrich die Ansiedlung von Algen untẹrschiedlich beeinflußte. Ein besonderes augenfälliges Beispiel dieser Art bot die zweimal beobachtete dichte Besiedelung schwarzer Buchstaben mit fädigen Grünalgen, während die weiße Grundfläche noch unbesiedelt war. Ein starker Bewuchs auf einer mit Überwasserfarbe gestrichenen Fläche konnte sich sehr scharf gegen den schwächeren Anwuchs auf der Unterwasserfarbe abgrenzen. Alle Bemühungen, diese Faktoren beweiskräftig aus dem übrigen Komplex herauszuschälen, scheiterten. Anzeichen für eine spezifische Farb-oder Giftwirkung auf die Ansiedelung bestimmter Arten wurden überhaupt nicht gefunden. Unabhängig von dem Nachweis einer Farbwirkung mußte natürlich mit ihrem Bestehen gerechnet werden, und nur Vergleiche zwischen gleichartig gestrichenen Flächen bzw. Tonnen sind völlig korrekt.

Abschließend wäre noch ein Wort über die Entwicklung des Bewuchses zu sagen. Direkte Beobachtungen fehlen, weil die meisten Tonnen nur einmal am Ende ihrer Liegezeit gut untersucht werden konnten. Der Vergleich von Tonnen mit verschieden langer Liegezeit ergab erwartungsgemäß, daß die Stärke des Anwuchses im Laufe der Zeit zunahm, weil die Pflanzen größer wurden und sich neue Algen ansiedelten. Das Bewuchsbild änderte sich aber auch im Laufe der Zeit, weil die Zahl der Arten größer wurde und sich ihr gegenseitiges Mengenverhältnis verschob. Die ersten makroskopisch erkennbaren Besiedler waren Diatomeen und Ectocarpus-Arten (vgl. z. B. Wilson 1925), die sich besonders an der Wasserlinie in großen Mengen ansiedeln konnten, im Laufe der Zeit aber von den nachfolgenden größeren Arten mehr oder weniger unterdrückt wurden. Dieser Vorgang verlief im Außengebiet, offenbar infolge der dort geringeren Zahl von Algensporen langsamer. Die größeren Arten wiederum wurden im Laufe der Zeit zum Teil von den Miesmuscheln verdrängt und von den Amphipoden verbaut (vgl. CASPERs, S. 150). Nur die Algen über und an der Wasserlinie sowie die sehr kräftigen Laminarien konnten widerstehen und bestimmten zusammen mit den Miesmuscheln das von uns beobachtete Endbild, das ähnlich oder gleichartig ausgeprägt oft an Schiffen und anderen schwimmenden Gegenständen beobachtet und untersucht wurde (vgl. z. B. Börgesen 1905, HentsChel 1923, 1924, Visscher 1930, Neu 1932, 1933, Fraser 1938). 


\section{Uberblick und Besprechung der Befunde}

Das Auftreten einer Art auf einer Boje setzt voraus, daß ihre Fortpflanzungskörper von einem Verbreitungsgebiet der Alge dorthin gelangen, sich ansiedeln und entwickeln können. Sind diese Bedingungen in demselben Maße am Ursprungs-' wie am Versuchsort erfüllt, so kann man erwarten, daß nach einer gewissen Zeit die Arten in annähernd gleicher Menge und Entwicklung vorhanden sind. Man darf also annehmen, daß im vorliegenden Falle Helgolands zumindest bei den Tonnen in Insel- und Dünennähe stets genügend Fortpflanzungskörper vorhanden sind und daher die ökologischen Verhältnisse das Bewuchsbild bestimmen. Man sollte also auf den Tonnen Algen der Supraund oberen Sublitoralzone, aber auch Algen der Litoralzone erwarten, sofern deren Vorkommen nicht an den Gezeitenwechsel als ökologischen Faktor gebunden ist.

Diese Erwartungen wurden auch in mancher Hinsicht bestätigt. Die auf den Tonnen reichlich vorkommenden Arten (Enteromorpha, Punctaria, Laminaria, Ceramium, Polysiphonia) sind auch um Helgoland gemein; umgekehrt traten die bei Helgoland seltenen Arten an den Tonnen gar nicht oder nur sehr wenig auf (vgl. auch Nienburg 1925). Ferner soll in diesem Zusammenhang das zonenförmige Vorkommen mancher Arten auf den Tonnen erwähnt werden, eine an den Küsten und gerade in der Gezeitenregion geläufige Erscheinung, die auch von MILNE (1940) an Tonnen festgestellt und näher untersucht wurde. Milne analysierte den Bewuchs auf einer Reihe von Tonnen, welche 12 bis 15 Monate lang in der Tamarmündung und flußaufwärts an Orten mit verschiedenen und im einzelnen sehr gut bekannten Umweltbedingungen auslagen. Er unterschied drei deutlich ausgeprägte Algenzonen (fädige Grünalgen, Enteromorpha-Ulva, Rotalgen-Laminaria), deren flußaufwärts abnehmende Breite er zudem mit der gleichzeitig gemessenen und infolge der Wassertrübung flußaufwärts zunehmenden Tageslichtabsorption gut korrelieren konnte. Außerdem wies er mit Nachdruck auf die auch von uns gefundene Tatsache hin, daß die Zonen an den Tonnen offenbar wegen der andersartigen Lebensverhältnisse längst nicht so breit wie in der Gezeitenregion waren.

Bemerkenswerter jedoch als die gemeinsamen Züge im Tonnenbewuchs und in der Helgoländer Algenflora erscheinen die Unterschiede. Am auffälligsten ist das Fehlen der bei Helgoland so häufigen Fucus-Arten, F. platycarpus, $F$. vesiculosus und $F$. serratus. Von den anderen Algen, die sonst bei Helgoland in der Gezeiten- oder oberen Sublitoralzone nicht eigentlich selten sind, aber nicht auf den Tonnen gefunden wurden, seien erwähnt die Krustenund Kalkalgen, Dictyota, Cladostephus, Cystoclonium, Chordaria, Ahnfeltia, Chondrus und Polyides. Andere bei Helgoland häufige Algen, wie z. B. Cladophora-Arten, kamen auf den Tonnen nur wenig und in schlechtem Zustand vor. Es liegt nahe, die Erklärung hierfür in der Besonderheit des untersuchten Lebensraumes - seiner gleichbleibenden Lage zum Wasserspiegel - zu suchen, also ungeeignete Lebensbedingungen besonders für die Algen der Gezeitenzone anzunehmen. Diese Vermutung wird sicherlich für viele der genannten Algen zutreffen, leuchtet aber für die sehr häufigen Fucus-Arten, und besonders für alle drei zusammen, als alleinige Ursache nicht unmittelbar ein. Platzmangel oder ungeeignete Lichtverhältnisse können jedenfalls nicht die wesentlichen Ursachen ihres Fehlens sein. Die Beobachtungen von Milne, der 
an Tonnen (junge) Fucus-Pflanzen und Ascophyllum fand, zeigen auch, daß geeignete Lebensbedingungen an Tonnen wenigstens in bestimmten Fällen für Fucus realisiert sein können. Nun ist ferner bekannt, daß Fucus-Pflanzen relativ langsam wachsen (vgl. z. B. NienBURG 1925) und von größeren Formen wie Ascophyllum stark unterdrückt werden können (vgl. z. B. Burrows and L.ODGE 1951), so daß man mit der Möglichkeit rechnen muß, daß junge FucusPflanzen bei den Untersuchungen übersehen wurden. Diese Möglichkeit erscheint aber angesichts der genauen Durchsicht vieler Proben im Laboratorium, die wenigstens gelegentlich einige Pflänzchen hätten ergeben sollen, recht unwahrscheinlich, so daß man - zumal auch auf Tonnen mit langer Liegezeit niemals eine Fucus-Pflanze gefunden wurde - bestrebt ist, nach einer Erklärung zu suchen, die nicht direkt mit den Lebensbedingungen zusammenhängt und auch der Tatsache Rechnung trägt, daß über das Vorkommen von Fucus-Pflanzen an schwimmenden Gegenständen im Gegensatz zu anderen häufigen Grün- und Braunalgen sehr selten berichtet wurde. Man könnte daran denken, daß die Eier der Fucales (und Dictyotales) viel größer und schwerer als die aller anderen Algen sind und, wie einfache Vergleichsversuche mit Suspensionen von Fucus-Eiern und Sporen oder Zygoten anderer Algen unmittelbar zeigen, bemerkenswert leicht auch bei bewegtem Wasser zu Boden sinken. Mit der Annahme, daß die Fucus-Eier nicht in -genügender Zahl an die Tonnen gelangen, soll nicht die Möglichkeit ausgeschlossen sein, daß nicht auch andere Faktoren zur Erklärung für das Fehlen von Fucus auf den beobachteten Tonnen herangezogen werden könnten, doch scheint sie uns zur Erklärung der bisherigen Befunde trotz aller Primitivität vorerst ausreichend zu sein.

Wie wichtig für die Besiedelung der Tonnen nicht nur die reichliche Verbreitung einer Art im Helgoländer Gebiet, sondern vor allem der Umstand ist, daß ihre Fortpflanzungskörper auch tatsächlich dorthin gelangen, zeigt die mit zunehmendern Abstand von der Insel allmählich abnehmende Besiedelung. Die geringen Unterschiede im Salzgehalt und in der Temperatur zwischen den einzelnen Tonnenpositionen können als solche wohl nicht die Bewuchsunterschiede erklären. Sie deuten aber darauf hin, daß Strömungen sicherlich an der geringeren bzw. langsameren Besiedelung der Mittel- und Außengebietstonnen beteiligt sind. Ihr Einfluß läßt sich angesichts der bei Helgoland so komplizierten und dazu noch vom Wind stark beeinträchtigten hydrographischen Verhältnisse schwer übersehen. Nach Hessen (1929) kann man in unserem Innen- und Mittelgebiet mit einer recht gleichmäßigen und guten Wasserdurchmischung rechnen, wohl auch noch bei Loreleybank und bei Düne 0/1. Das Steingrund-Gebiet dagegen (vgl. ZoRELl 1935) liegt schon vorwiegend im Bereich des nordfriesischen Küstenwassers, so daß man zur Erklärung der Unterschiede des Bewuchses, die zwischen den Steingrund-Tonnen und allen übrigen bestehen, auch Strömungsfaktoren hinzuziehen muß. Wesentlich wird immer die Konzentration an lebensfähigen Keimen sein, die sich mit zunehmender Entfernung von der Insel verringert.

\section{E. Zusammenfassung}

Der im Verlauf zweier Jahre untersuchte Algenbewuchs der Fahrwassertonnen um Helgoland bestand aus Algen der Gezeiten-, Supra- und oberen 
Sublitoralzone. Thre Häufigkeit entsprach durchweg den Helgoländer Verhältnissen, es fehlten jedoch die drei bei Helgoland sehr reichlich vorkommenden Fucus-Arten ( $F$. platycarpus, $F$. vesiculosus und F. serratus). Einige Algen (fädige Grünalgen, Bangia, Enteromorpha, Punctaria, Ceramium, Polysiphonia und Laminaria) konnten in gürtelförmigen Zonen auftreten; diese sind schmäler als am natürlichen Standort. Der Algenanwuchs verringert sich mit zunehmender Entfernung von der Insel.

\section{Literatur}

Börgesen, F., 1905. The algae-vegetation of the Færöese coasts. Botany of the Færöes 3. Kopenhagen.

Burrows, E. M. and Lodge, Sh., 1951. Autecology and the species problem in Fucus. J. Mar. Biol. Ass. U. K. 30.

Caspers, H., 1952. Der tierische Bewuchs an Helgaländer Seetonnen. Helgol. Wiss. Meeresunters. 4.

Evans, R. G., 1947. The intertidal ecology of selected localities in the Plymouth neighbourhood. Ebenda 27.

Fraser, J. H., 1938. The fauna of fixed and floating structures in the Mersey Estuary and Liverpool bay. Proc. Liverpool Biol. Soc. 51.

Goedecke, E., 1939. Beitrag zur Hydrographie der Helgoland umgebenden Gewässer Ann. Hydrogr. Marit. Meteorol. 67.

Hagmeier, A., 1930. Die Besiedelung des Felsstrandes und der Klippen von Helgoland. Teil 1. Der Lebensraum. Wiss. Meeresunters. N. F. Abt. Helgol. 15.

Hatton, H., 1938. Essais de bionomie explicative sur quelques espèces intercotidiales d'algues et d'animaux. Ann. Inst. Océanogr. 17.

Hentschel, E., 1923. Der Bewuchs an Seeschiffen. Int. Revue ges. Hydrobiol. u. Hydrogr. 11.

- 1924. Das Werden und Vergehen des Bewuchses an Schiffen. Mitt. Zool. Staatsinst. u. Zool. Mus. Hamburg 41.

Hessen, K., 1929. Karten der Strömungen in der Nähe von Helgoland. Marinewerft Wilhelmshaven.

Kirchenpauer, G. H., 1862. Die Seetonnen der Elbmündung. Abh. a. d. Gebiete d. Naturw., herausg. v. Natw. Ver. Hamburg 4.

Kitching, J. A., 1935. An introduction to the Ecology of intertidal rock surfaces on the coast of Argyll. Transact. Roy. Soc. Edinburgh 58.

Knight, M. and Parke, M., 1950. A biological study of Fucus vesiculosus L. and F. serratus L. J. Mar. Biol. Ass. U. K. 29.

Kuckuck, P., 1894. Bemerkungen zur marinen Algenvegetation von Helgoland. Wiss. Meeresunters., N. F., Abt. Helgol. 1.

- 1897. Uber marine Vegetationsbilder. Ber. D. Bot. Ges. 15.

Milne, A., 1940. The Ecology of the Tamar Estuary. IV. The distribution of the Fauna and Flora on buoys. J. Mar. Biol. Ass. U, K. 24.

Nienburg, W., 1925. Die Besiedelung des Felsstrandes und der Klippen von Helgoland. Teil II: Die Algen. Wiss. Meeresunters., N. F., Abt. Helgol. 15.

- 1930. Die festsitzenden Pflanzen der nordeuropäischen Meere. In Ehrenbaum und Lübbert, Handb. d. Seefischerei Nordeuropas. Bd. I, Heft 4, Stuttgart.

$\mathrm{Neu}$, W., 1932. Untersuchungen über den Schiffsbewuchs. Int. Revue ges. Hydrobiol. u. Hydrogr. 27.

- 1933. Qualitative und quantitative Bewuchsuntersuchungen am Elbe-Feuerschiff "Bürgermeister Bartels" 1932. Arch. Hydrobiol. 25.

Visscher, J. P., 1928. Nature and Extent of Fouling of Ship's Bottom. Bull. Bureau of Fischeries, Washington 43, Part II.

Wils on, O. T., 1925. Some experimental observations of marine algal successions. Ecology 6.

Zore11, Fr., 1935. Beiträge zur Hydrographie der Deutschen Bucht. Aus dem Archiv der Deutschen Seewarte, Hamburg 54. 